\title{
SCATTERING PROPERTIES OF ASYMMETRIC RECTANGULAR IRIS IN A CIRCULAR WAVEGUIDE USING EDGE-CONDITIONED BASIS FUNCTIONS
}

\author{
T. Lenadan, S. Amari, R. Vahldieck and J. Bornemann \\ Department of Electrical and Computer Engineering \\ University of Victoria, Victoria B.C., Canada V8W 3P6
}

\section{Abstract}

The scattering properties of asymmetric rectangular irises in circular waveguides are accurately determined from an integral equation formulation using basis functions which include the edge conditions. The inclusion of the edge conditions results in a reduction of the size of the matrix as compared to the standard Mode-Matching Technique. Numerical results are presented and compared with previously published data and measurements to demonstrate the accuracy of the approach.

\section{INTRODUCTION}

Coupling irises are frequently used in modern waveguide filters and matching networks whose design relies, to a large extent, on the accurate prediction of the scattering properties of their different components.

The Mode-Matching Technique (MMT) is commonly used to determine the generalized scattering matrices of the individual discontinuities in these devices [1]. It is, however, known that the MMT converges slowly because of its failure to take into account the singular nature of the elec- tromagnetic field at the sharp metallic corners. A large number of modes are often required to attain acceptably accurate results for the overall scattering characteristics. Despite the fact that, in most applications, only the fundamental mode is propagating in the structure, the MMT extracts the scattering properties from a generalized scattering matrix which contains higher order modes.

The scattering of the fundamental mode $T E_{11}$ of a circular waveguide from a rectangular iris of finite thickness was investigated by Macphie and $\mathrm{Wu}$ using the MMT [2]. To improve the numerical implementation of the technique, the coupling integrals were computed analytically by expanding cylindrical functions (Bessel functions) in terms of plane waves [2]. The edge conditions of the electromagnetic fields were not included in the analysis.

In this paper, we propose to determine the scattering properties of asymmetric rectangular irises in a circular waveguide from an integral equation for the transverse electric field at the discontinuity using basis functions which include the edge conditions. The expansion of Bessel functions in series of plane waves presented in [2] is also used in this work to compute the matrix elements which appear when Galerkin's method 
is implemented. Using this approach we concentrate directly on the transmission and reflection of the incident modes at the input and output while taking into account the fact that higher order modes are excited only at the discontinuities.

\section{THEORY}

The generalized scattering matrix of a rectangular iris of finite thickness is determined from cascading the individual scattering matrices of the two circular-to-rectangular discontinuities. We therefore concentrate on the analysis of a circular-to-rectangular step discontinuity whose cros-section is depicted in figure 1 . To derive an

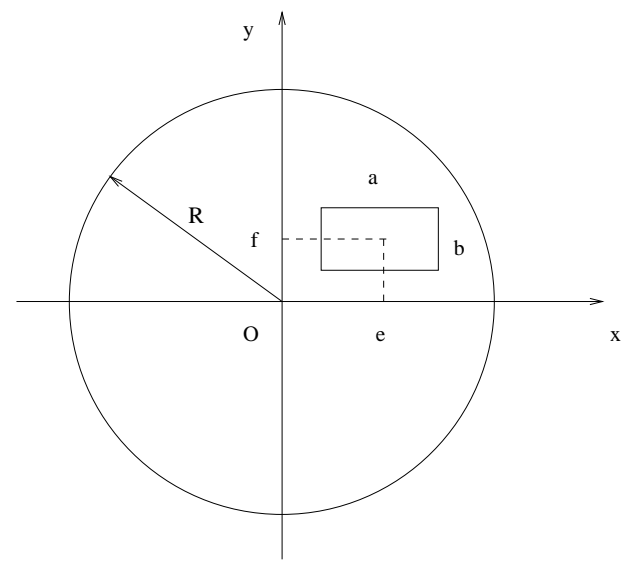

Figure 1: Cross section of the junction

integral equation for the transverse electric field at the discontinuity, the electromagnetic field in regions $\mathrm{I}$ and II is expanded in modal series of the TE and TM modes of the corresponding regions.

From the boundary conditions of the transverse electric field at the discontinuity, and using the orthogonality of the modes in the two regions, the modal expansion coefficients are expressed in terms of weighted integrals of the transverse electric field, which we denote by $\mathbf{Z}(x, y)$, over the cross section of the rectangular waveguide. These resulting expressions of the modal coefficients are then substituted in the continuity condition of the magnetic field to obtain an integral equation for $\mathbf{Z}(x, y)$.

By formulating the problem in terms of the electric field at the discontinuity, it is now possible to include in the theory whatever a priori information we have on the exact solution such as the edge conditions. We therefore expand $\mathbf{Z}(x, y)$, or its $\mathrm{x}$ - and $\mathrm{y}$-components, in series of basis functions which include the edge conditions. Galerkin's method is then applied to the integral equation to determine the expansion coefficients of $\mathbf{Z}(x, y)$.

Once function $\mathbf{Z}(x, y)$ is determined, the modal expansions of the electromagnetic field, and therefore the reflected and transmitted waves, are fully specified in each of the two regions of the structure. The details of the approach as applied to $\mathrm{H}$-plane discontinuities in rectangular waveguides can be found in [3].

\section{BASIS FUNCTIONS}

To guarantee numerical efficiency, the basis functions should include the singular nature of the transverse electric field at the sharp metallic edges of the discontinuity. In the present situation, the component of the electric field perpendicular to the metallic wedge becomes infinite as $r^{-1 / 3}$ where $r$ is the radial distance form the wedge whereas the component parallel to the wedge vanishes as $r^{2 / 3}$ as $r \rightarrow 0$ [4]. The following set of basis functions were used in this work

$$
B_{m n}^{x}(x, y)=\frac{\frac{\cos \left[m \pi \frac{x-a / 2-e}{a}\right]}{[(x+a / 2-e)(a / 2+e-x)]^{1 / 3}} \times}{\frac{\sin \left[n \pi \frac{y+b / 2-f}{b}\right]}{[(y+b / 2-f)(b / 2+f-y)]^{1 / 3}}}
$$


and

$$
\begin{aligned}
B_{m n}^{y}(x, y)=\frac{\sin \left[m \pi \frac{x-a / 2-e}{a}\right]}{[(x+a / 2-e)(a / 2+e-x)]^{1 / 3}} \times \\
\frac{\cos \left[n \pi \frac{y+b / 2-f}{b}\right]}{[(y+b / 2-f)(b / 2+f-y)]^{1 / 3}}
\end{aligned}
$$

The spectrum of these basis functions in the rectangular waveguide can be expressed in terms of Bessel functions of order 1/6 [5]. In the circular waveguide, a series expansion of Bessel functions in plane waves, as discussed in [2], is used to express the spectrum of the basis functions also in terms of Bessel functions of order 1/6.

\section{$4 \quad$ RESULTS}

The scattering of the fundamental mode in a circular waveguide at a rectangular-to-circular discontinuity as well as a rectangular iris of finite thickness was investigated using the present technique. Figure 2 shows the insertion and re-

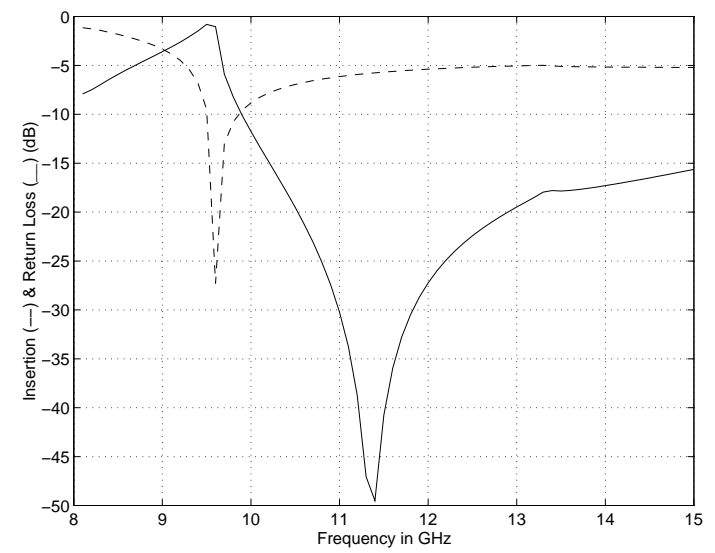

Figure 2: Insertion and return loss in $\mathrm{dB}$ of the circular to rectangular junction $(\mathrm{a}=2 \mathrm{~b}=$ $19.05 \mathrm{~mm}, \mathrm{R}=\mathrm{a}, \mathrm{e}=0$ and $\mathrm{f}=0$ ).

turn losses of a centered transition from circular waveguide to smaller rectangular waveguide as a function of frequency. Our results agree well with those presented in reference [2] (not shown here). The agreement between the two computations is excellent over the entire range of frequencies. These results where obtained using 12 basis functions for each of the components $E_{x}$ and $E_{y}$. The number of basis functions necessary was determined from a convergence analysis as shown in figure 3. Figure 4 shows the in-

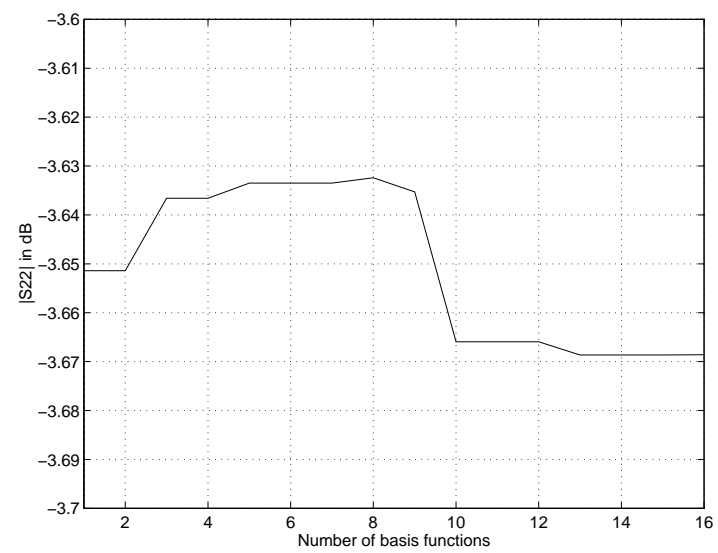

Figure 3: Convergence of $-S_{22}$ - versus the number of basis functions when $98 \mathrm{TE}, 49 \mathrm{TM}$ circular modes and 14 TE, 7 TM rectangular modes are used $(\mathrm{F}=9 \mathrm{GHz})$.

sertion and return losses of the circular to off-set rectangular junction as a function of frequency. Our results agree well with those presented in reference [2] (not shown here) which attests to the validity of the approach.

The insertion and return losses of a rectangular iris of length $\mathrm{L}=0.1 \mathrm{~mm}$ where also computed and are shown in figure 5. These results were obtained using 10 basis functionsfor each of the components $E_{x}$ and $E_{y}$.

\section{CONCLUSIONS}

An integral-equation approach was used to determine the scattering properties of a circular- 


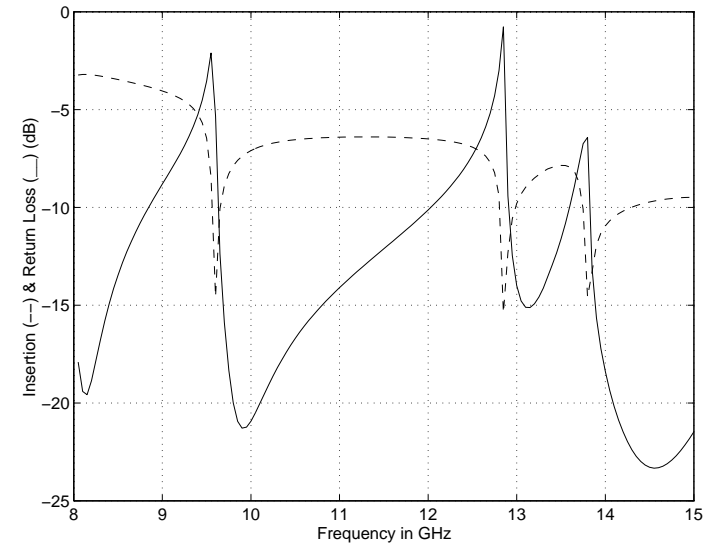

Figure 4: Insertion and return loss in $\mathrm{dB}$ of the circular to off-set rectangular junction $(\mathrm{a}=2 \mathrm{~b}=19.05 \mathrm{~mm}, \mathrm{R}=\mathrm{a}, \mathrm{e}=6.35 \mathrm{~mm}, \mathrm{f}=$ $4.72 \mathrm{~mm})$

to-rectangular discontinuity as well as a rectangular iris of finite thickness in a circular waveguide. Basis functions which include the edge conditions were used to accelerate convergence and reduce the size of the matrices. The coupling integrals are computed analytically from an expansion of Bessel functions in series of plane waves. Results obtained from the present technique agree well with previously published data.

\section{REFERENCES}

1. T. Itoh ed., Numerical Techniques for Microwave and Millimeter-Wave Passive Structures, John Wiley \& Sons, New York 1989.

2. R. H. Macphie and Ke-Li Wu, "Scattering at the junction of a rectangular waveguide and a larger circular waveguide," IEEE Trans. Microwave Theory Tech., vol. 43, pp. 2041-2045, Sept. 1995.

3. S. Amari, J. Bornemann and R. Vahldieck,

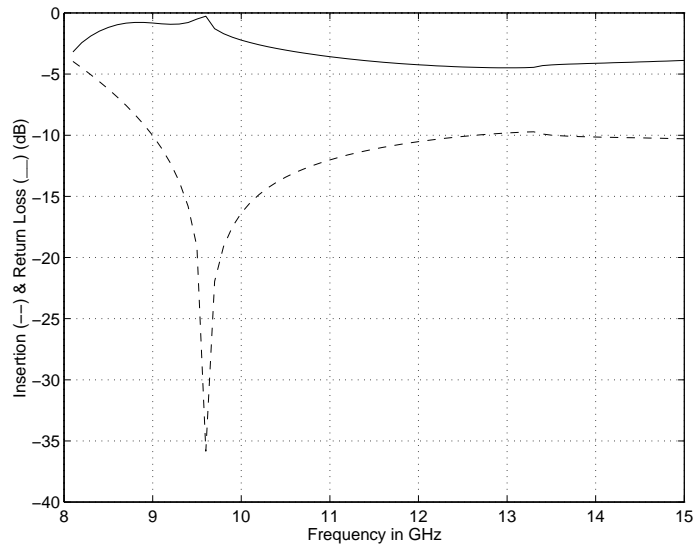

Figure 5: Insertion and return loss versus frequency of a rectangular iris in a circular waveguide $(\mathrm{a}=2 \mathrm{~b}=19.05 \mathrm{~mm}, \mathrm{R}=\mathrm{a}$ and $\mathrm{L}=0.1 \mathrm{~mm})$

"Accurate analysis of scattering from multiple waveguide discontinuities using the coupledintegral-equations technique," Jour. Electromagnetic Waves and Applications, vol. 10, pp. 1623-1644, Dec. 1996.

4. R. E. Collin, Field Theory of Guided Waves, IEEE Press, New York, 1991.

5. I. S. Gradshteyn and I. M. Ryznik, Tables of Integrals, Series, and Products, Fifth Edition, Academic Press, New York, 1994. 